\title{
Effect of Fast Shift Rotation and Slow Shift Rotation on the Nurses' Fatigue and Sleep Quality
}

\author{
Minping Deng ${ }^{1}$, Weiju Chen ${ }^{2,}$, Tianying Pang ${ }^{1}$, Chunmei Lin ${ }^{1}$ \\ ${ }^{1}$ Gastrointestinal Surgical Unit, The First Affiliated Hospital, Jinan University, Guangzhou, China \\ ${ }^{2}$ Department of Nursing, The First Affiliated Hospital, Jinan University, Guangzhou, China
}

Email address:

dengminping@126.com (Minping Deng),weijuchen2008@163.com (Weiju Chen)

*Corresponding author

\section{To cite this article:}

Minping Deng, Weiju Chen, Tianying Pang, Chunmei Lin. Effect of Fast Shift Rotation and Slow Shift Rotation on the Nurses' Fatigue and Sleep Quality. American Journal of Health Research. Vol. 7, No. 2, 2019, pp. 19-23. doi: 10.11648/j.ajhr.20190702.11

Received: April 25, 2019; Accepted: June 3, 2019; Published: June 12, 2019

\begin{abstract}
Objective: The objective of this study was to investigate whether the three-shift fast rotation, two-shift fast rotation and night-shift slow rotation led to any differences in fatigue and sleep quality. Method: The study was conducted as a beforeafter study and 17 nurses participated in the study. Nurses on three-shift fast rotation were allocated to the pre-group, and two-shift fast rotation and night-shift slow rotation were allocated to the post-group. In the study period, all of the subjects experienced the three patterns of rotation. Occupational Fatigue Exhaustion/Recovery Scale (OFER15) and Athens Insomnia Scale were used to measure the participants' work-related fatigue and sleep quality three times. Results: Athens Insomnia Scale scores showed significant differences between the three types of shift rotation; two-shift fast rotation was $3.88 \pm 2.35$, three-shift fast rotation was $8.63 \pm 3.50$, and night-shift slow rotation was $13.75 \pm 4.23(\mathrm{P}<0.05)$. Therefore, the nurses' sleep quality through working on the two-shift rotation was better than the other two types of rotation. However, there were no significant differences in chronic fatigue, acute fatigue, and inter-shift [recovery] between the three types of rotations. Conclusion: Nurses who worked on the two-shift fast rotation had a better sleep quality than those who worked on the three-shift fast rotation and night-shift rotations. As sleep quality relates to the performance and alertness of nurses, greater attention should be paid to the use of different shift combinations to increase sleep quality and alertness. Considering sleep quality, a three-shift system arranged by two-shift fast rotation and combined with night-shift slow rotation is superior to the three-shift fast rotation.
\end{abstract}

Keywords: Shift Rotation, Fast Shift Rotation, Slow Shift Rotation, Nurses' Fatigue, Sleep Quality

\section{Introduction}

Nearly $20 \%$ of the labor force worldwide work shifts which include work hours outside of 7 am to 18:00 pm [1]. Shift work, which disrupts the body's circadian rhythm and affects health, has been identified as an important source of occupational stress. The most common health-related effect of shift work is disturbed sleep, such as difficulty falling asleep and a shortened sleep duration [2]. Increasing evidence indicates that shift work schedules negatively influence worker physiology and health, for example they can result in circadian disruptions that are associated with cardiometabolic diseases, gastrointestinal health, and the worsening of mood disorders and depressive symptoms [3, 4]. Healthcare work is characterized by 24 -hour operations, so that drawing upon shift work is common for several nursing services. Shift work can be organized around fixed or rotating patterns. The fixed-shift pattern is where the employee works evenings or nights on a continuing basis and a rotated-shift pattern is where the employee may alternate between day, evening and night-shifts, or they may rotate between evening and nights [5] Studies have shown that fixed shifts may increase the likelihood that workers will, with time, adapt to their shift schedules by adjusting their sleeping patterns, eating behavior, family life, and social life [5]. Other studies have reported that permanent fixed night workers generally report poorer health, more absenteeism, and less job satisfaction than fixed day workers [6]. Rotating shifts, if done on a weekly or monthly basis, may avoid some of the problems associated with fixed nights and reduce the likelihood of a worker ever becoming fully adjusted to a particular shift [5]. Studies have shown that 
rotating shifts were significantly associated with acute fatigue, but when nurses work on schedules which are fixed on either days or nights, they can adapt to their schedule and may find successful strategies to reduce fatigue and increase recovery [7]. Furthermore, some studies have reported that nurses had a decrease in their perceptual and motor abilities on the three-shift system with fast rotation, suggesting that a fast rotating shift schedule may increase the risk of medical errors [8]. While many studies have investigated the influence of rotating shifts on night performance and sleep, little research has been performed on the influence of a three-shift fast rotation, two-shift fast rotation and night-shift slow rotation on sleep quality and work-related fatigue. In the authors' hospital, most nurses work on a three-shift system ( 8 hour shifts); two consecutive day shifts ( 8 am-15:30pm or 8 am18:00 pm), two evening shifts (15:30 pm-22:30 pm), one night-shift (22:30-8 am), and then have at least 1 day off duty before restarting the cycle. Previous research showed that nurses who rotated with the fast three-shift system had a decrease in their perceptual and motor abilities on the night-shift. To reduce the disadvantage of a fast-rotating pattern, the hospital has to be encouraged to apply the alternative shift or combine other shift rotating patterns. This study investigated whether the three-shift fast rotation, two-shift fast rotation, and night-shift slow rotation led to any differences in fatigue and sleep quality.

\section{Method}

\subsection{Participants and Procedures}

This study was conducted in the surgical wards of The First Affiliated Hospital of Jinan University, Guangzhou, China. Participants were female nurses who provided direct care for patients, worked full-time schedules (7-10 hours per shift), and worked different shifts including day shifts ( 7 hours), evening shifts ( 7 hours), and night-shifts (10 hours). We excluded from the study nurses who had taken hypnotic drugs, sedatives, anti-inflammatory drugs, beta blockers, or antihistamines within four weeks of the study. In addition to those who had neurological diseases, metabolic diseases, renal diseases, and mental disorders. A total of 17 nurses met the selection criteria. The researcher explained the theme, purpose, study content, study time, methods, and protection of fundamental rights of the study to participants. After this explanation, 17 nurses agreed to participate. This study was conducted as a before-after study. Three-shift fast rotation were allocated to pre-group, and two-shift fast rotation and night-shift slow rotation were allocated to post-group. In the study period, all of the subjects experienced the three patterns of rotation.

For the three-shift fast rotation pattern, nurses were rotated on morning, evening, and night-shifts for one week, usually arranged on two day shifts, two evening shifts, one night-shift, and then had at least one day off duty before restarting the cycle. All the participants began from the day shift. For the two-shift fast rotation pattern, nurses were rotated on the morning and evening shifts for one week, which were usually arranged as four day shifts, one day off, two evening shifts, and then at least one day off duty before restarting the cycle. All the participants began from the day shift. For the night-shift slow rotation pattern, nurses worked three-four nights, followed by four-three days off duty in one week.

\subsection{Measurement}

Sel-administered structured questionnaires were completed consisting of the following instruments: Basic sociodemographic data questionnaire, Athens Insomnia Scale, and Occupational Fatigue Exhaustion/Recovery Scale (OFER15).

\subsubsection{Athens Insomnia Scale (AIS)}

The Chinese version of the Athens Insomnia Scale (AIS) was used in this study to measure the participants' sleep quality over the past month. AIS is considered to be an effective tool in sleep analysis, and it is validated in various countries following testing on local patients [9-11]. AIS is an eight-factor self-reported questionnaire, where the first five factors are related to nocturnal sleep and last three factors are related to daytime dysfunction. These are rated on a $0-3$ scale and the sleep quality is finally evaluated from the cumulative score of all of the factors and reported as an individual's sleep outcome. Over the period of time, a cut-off score of $\geq 6$ on the AIS was used to establish the diagnosis of insomnia $[12,8]$.

\subsubsection{Occupational Fatigue Exhaustion/Recovery Scale}

The Chinese version of the Occupational Fatigue Exhaustion/Recovery Scale (OFER15) was used in this study to measure participants' work-related fatigue. OFER15 scale is composed of 15 items distributed on three subscales, each subscale consists of five items. Each item is rated with seven points on the Likert scale from " 0 " (strongly disagree) to " 6 " (strongly agree) [13]. This scale has been tested in different populations and has strong psychometric properties [14]. The Chinese version OFER's scale demonstrated high construct and face validity, and its three subscales showed good internal consistency reliability. Therefore, it is a reliable and valid instrument for assessing work-related fatigue in Chinese nurses [15].

\subsection{Data and Statistical Analysis}

The participants were surveyed 3 times: the first time was on the last day of the one month three-shift fast rotation period; the second time was on the last day of the one month two-shift fast rotation period; and the last time was on the last day of the one month night-shift slow rotation. AIS and OFER15 questionnaires were established online, and participants were invited to finish the questionnaire on the last day of each rotation pattern. Data collected were anonymous and completely confidential and were used only for academic research. All data were entered and analyzed using SPSS 13.0. Sleep quality and work-related fatigue were described using the descriptive statistics including mean and standard deviation. Pearson's correlation was used to examine the 
relationships between the independent variables (shift rotation types) and the dependent variables (sleep quality and fatigue). The findings were significant at a value of $\mathrm{P}<0.05$.

\section{Results}

\subsection{Nurses'Sleep Quality Between the Three Types Shift Rotation}

AIS scores showed significant differences between the three types of shift rotation; two-shift fast rotation was $3.88 \pm$ 2.35 , three-shift fast rotation was $8.63 \pm 3.50$, and night-shift slow rotation was $13.75 \pm 4.23(\mathrm{P}<0.05)$. Therefore, the nurses' sleep quality when working on the two-shift rotation was better than the other two types of rotation. The eight-factor scores are shown in Table 1, and the total sleep duration, sleep quality, well-being during the day, functioning capacity during the day, and sleepiness during the day showed significant differences between the shifts.

Table 1. Eight-factor scores of AIS in three types of shift rotation.

\begin{tabular}{lllll}
\hline & $\begin{array}{l}\text { Three-shift fast } \\
\text { rotation (Means) }\end{array}$ & $\begin{array}{l}\text { Two-shift fast } \\
\text { rotation (Means) }\end{array}$ & $\begin{array}{l}\text { Night-shift slow } \\
\text { rotation (Means) }\end{array}$ & F \\
\hline Sleep induction & 0.88 & 0.63 & 1.25 & 1.72 \\
Awakenings during the night & 0.88 & 0.75 & 1.30 & 1.27 \\
Final awakening & 0.5 & 0.5 & 1.25 & 3.31 \\
Total sleep duration & 1.88 & 0.50 & 1.25 & 12.01 \\
Sleep quality & 1.83 & 0.63 & 1.20 & 4.83 \\
Well-being during the day & 0.38 & 0.24 & 1.88 & 0.68 \\
Functioning capacity during the day & 1.38 & 0.13 & 2.38 & 0.00 \\
Sleepiness during the day & 1.13 & 0.75 & 2.63 & 0.01 \\
\hline
\end{tabular}

\subsection{Work-Related Fatigue Between the Three Types Shift Rotation}

Work-related fatigue scores showed no significant differences in chronic fatigue, acute fatigue, and inter-shift [recovery] between the three types of rotation.

Table 2. Work-related fatigue sores between the three types shift rotation $(x \pm s)$.

\begin{tabular}{lllll}
\hline & Three-shift fast rotation & Two-shift fast rotation & Night-shift slow rotation & F \\
\hline Chronic fatigue & $33.33 \pm 17.72$ & $34.17 \pm 11.65$ & $36.66 \pm 18.60$ & 0.09 \\
Acute fatigue & $48.33 \pm 12.84$ & $41.25 \pm 17.82$ & $46.25 \pm 14.08$ & 0.91 \\
Inter-shift [recovery] & $46.66 \pm 4.71$ & $43.75 \pm 12.00$ & $45.41 \pm 9.58$ & 0.63 \\
\hline
\end{tabular}

\section{Discussion}

Shift work has significantly changed nurses' sleep patterns, between $57 \%$ and $83.2 \%$ [16, 17]. Poor sleep quality and insufficient sleep have an injurious effect on both the health and performance of nurses [18]. The results of this study suggest that there were significant differences in sleep quality between three different types of shift rotation, as measured by AIS. Nurses with the best sleep quality worked a two-shift rotation and on the other two types of shift rotation (three-shift fast rotation and night-shift rotation), nurses were diagnosed with insomnia (AIS scores $>6$ ). Prior research has reported that insufficient sleep and poor sleep quality impact metabolism, the immune system, and the digestive system resulting in psychophysiological health problems such as emotional disturbances [19, 20]. Other studies have shown that the better the sleep at night, the greater the daytime alertness [21], meaning that in this study daytime alertness was higher when nurses were on the two-shift fast rotation pattern as opposed to the other two. Five of the eight factors of the OFER15 scale showed significant differences. Rotating shifts change frequently from day to night-shifts and most nurses working night-shifts struggle to adjust to daytime activities or normal night sleep patterns on their days off [18]. In this study, nurses had the best total sleep time and daytime performance on the two-shift fast rotation pattern, followed by the three-shift fast rotation and then the night-shift slow rotation patter. As the two-shift fast rotation pattern rotated between morning and evening shifts and did not include night-shifts, it had less interference on the circadian rhythm. Nurses reported less well-being and more sleepiness during the day when on the night-shift slow rotation pattern. However, these two factors were asked with regard to the daytime situation, but as the daytime was the rest time for the nurses on this type of rotation led to this result.

Work-related fatigue is one of the most common phenomena among shift workers, particularly nurses [22]. Studies found that $91.9 \%$ of nurses complained of work-related fatigue with a higher level among female nurses [23]. Concerning fatigue, the study revealed that in an eight hour shift system there were no significant differences in fatigue (chronic fatigue, acute fatigue, and inter-shift [recovery]) between the three types of rotations as measured by the Occupational Fatigue Exhaustion/Recovery Scale, but the study had a lower average score than other studies [22]. The result is supported by previous studies which showed no significant relationship between the number of night-shifts and fatigue [24, 25]. Furthermore, other previous studies have reported that the number of night and evening shifts per month had indirect effects on both acute and chronic fatigue, as well 
as on inter-shift recovery [26]. The study findings also indicated that the scores of chronic and acute fatigue, which are inconsistent with a previous study [27], were lower than the mentioned studies and might be interpreted differently due to the working hours; in this study nurses worked $7-10$ hours per shift. Furthermore, some studies have documented nurses working daily shifts of 8-9 or 9-10 hours reported lower physical fatigue than nurses working daily shifts of $10-11$ or more than 12 hours [28].

\section{Conclusion}

The findings of this study indicate that in the three-shift system, the nurses who worked on the two-shift fast rotation had a better sleep quality than those who worked on the three-shift fast rotation and night-shift rotation. As sleep quality is related to the performance and alertness of nurses, greater attention should be paid to different shift combinations with a view to increasing the sleep quality and alertness. Different combinations of the three-shift system had different effects on the sleep quality of nurses. Nurses on the three-shift fast rotation (rotated morning, evening, and nights in one week) had affected sleep quality and were diagnosed with insomnia. However, when the two-shift fast rotation was combined with the night-shift slow rotation pattern, sleep quality was effected only when the nurses were in the night-shift slow rotation period (one month), and there were no effects on the sleep quality of the nurses on the two-shift fast rotation period. Considering sleep quality, a three-shift system arranged by two-shift fast rotation and combined with a night-shift slow rotation, is superior to the three-shift fast rotation.

\section{Funding}

Funding by Medical Scientific Research Foundation of Guangdong Province of China, Number: B2018046.

\section{Conflict of Interest}

All authors declare that they have no conflicts of interest.

\section{References}

[1] Wright KP, Jr., Bogan RK, Wyatt JK: Shift work and the assessment and management of shift work disorder (swd). Sleep medicine reviews 2013; 17: 41-54.

[2] Burch JB, Tom J, Zhai Y, Criswell L, Leo E, Ogoussan K: Shiftwork impacts and adaptation among health care workers. Occupational medicine 2009; 59: 159-166.

[3] Davila EP, Florez H, Fleming LE, Lee DJ, Goodman E, Leblanc WG, Caban-Martinez AJ, Arheart KL, Mccollister KE, Christ SL: Prevalence of the metabolic syndrome among u. S. Workers. Diabetes Care 2010; 33: 2390-2395.

[4] Gu F, Han J, Laden F, Pan A, Caporaso NE, Stampfer MJ, Kawachi I, Rexrode KM, Willett WC, Hankinson SE: Total and cause-specific mortality of $u$. S. Nurses working rotating night shifts. American journal of preventive medicine 2015; 48: 241-252.

[5] Perrucci R, MacDermid S, King E, Tang C-Y, Brimeyer T, Ramadoss K, Kiser SJ, Swanberg J: The significance of shift work: Current status and future directions. Journal of Family and Economic Issues 2007; 28: 600-617.

[6] Burch JB, Tom J, Zhai Y, Criswell L, Leo E, Ogoussan K: Shiftwork impacts and adaptation among health care workers. Occup Med 2009; 59: 159-166.

[7] Han K, Trinkoff AM, Geiger-Brown J: Factors associated with work-related fatigue and recovery in hospital nurses working 12-hour shifts. Workplace health \& safety 2014; 62: 409.

[8] Yu SC, Yu HW, Hsu CY, Shu HT, Li LY, Shu FS: Impairment of perceptual and motor abilities at the end of a night shift is greater in nurses working fast rotating shifts. Sleep medicine 2011; 12: 866-869.

[9] Jia-Ling S, Jeng-Fong C, Chia-Chin L: Validation of the taiwanese version of the athens insomnia scale and assessment of insomnia in taiwanese cancer patients. J Pain Symptom Manage 2011; 41: 904-914.

[10] Isa O, Shun N, Mina K, Yuichi I: Development and validation of the japanese version of the athens insomnia scale. Psychiatry \& Clinical Neurosciences 2013; 67: 420-425.

[11] Paparrigopoulos T, Tzavara C, Theleritis C, Psarros C, Soldatos $\mathrm{C}$, Tountas Y: Insomnia and its correlates in a representative sample of the greek population. BMC public health 2010; 10: 531 .

[12] Soldatos CR, Dikeos DG, Paparrigopoulos TJ: The diagnostic validity of the athens insomnia scale. Journal of Psychosomatic Research 2003; 55: 263-267.

[13] Winwood PC, Lushington K, Winefield AH: Further development and validation of the occupational fatigue exhaustion recovery (ofer) scale. Journal of Occupational \& Environmental Medicine 2006; 48: 381-389.

[14] Winwood PC, Winefield AH, Dawson D, Lushington K, Development and validation of a scale to measure work-related fatigue and recovery: The occupational fatigue exhaustion/recovery scale (ofer). Journal of Occupational \& Environmental Medicine 2005; 47: 594-606.

[15] Fang JB, Zhou CF, Huang J, Qiu CJ: Psychometric properties of the chinese version of the occupational fatigue exhaustion/recovery scale: A test in a nursing population. Journal of Nursing Research 2018; 26 : 1.

[16] Shao MF, Chou YCYeh MY, Tzeng WC: Sleep quality and quality of life in female shift-working nurses. Journal of advanced nursing 2010; 66: 1565-1572.

[17] Roodbandi AJ, Choobineh A, Daneshvar S: Relationship between circadian rhythm amplitude and stability with sleep quality and sleepiness among shift nurses and health care workers. International Journal of Occupational Safety \& Ergonomics 2015; 21:312-317.

[18] Sun Q, Ji X, Zhou W, Liu J: Sleep problems in shift nurses: A brief review and recommendations at both individual and institutional levels. Journal of nursing management 2019; 27: 10-18. 
[19] Korsiak J, Tranmer J, Day A, Aronson KJ: Sleep duration as a mediator between an alternating day and night shift work schedule and metabolic syndrome among female hospital employees. Occupational \& Environmental Medicine 2018; 75: 132.

[20] Lee C, Chen H, Meg MT, Lee H, Huang L: The relationships among sleep quality and chronotype, emotional disturbance, and insomnia vulnerability in shift nurses. Journal of Nursing Research 2015; 23: 225-235.

[21] Kayumov L, Rotenberg V, Buttoo K, Auch C, Pandi-Perumal SR, Shapiro CM: Interrelationships between nocturnal sleep, daytime alertness, and sleepiness: Two types of alertness proposed. Journal of Neuropsychiatry \& Clinical Neurosciences 2000; 12: 86 .

[22] Ismail KM, Malak MZA-Ohoo, Alamer RM: Psychosocial correlates of work-related fatigue among jordanian emergency department nurses. Lid - 10.1111/ppc.12354 [doi]. 2019.

[23] Raftopoulos V: The factors associated with the burnout syndrome and fatigue in cypriot nurses: A census report. BMC public health 2012; 12: 457-457.

[24] Elisabeth F, St?Le P, Bente Elisabeth M, Siri W, Bj?Rn B: Short rest periods between work shifts predict sleep and health problems in nurses at 1-year follow-up. Occupational \& Environmental Medicine 2014; 71: 555-561.

[25] Jung HS, Lee B: Contributors to shift work tolerance in south korean nurses working rotating shift. Applied Nursing Research 2015; 28: 150-155.

[26] Fang J, Qiu C, Xu H, You G: A model for predicting acute and chronic fatigue in chinese nurses. Journal of advanced nursing 2013; 69: 546-558.

[27] Sagherian K, Clinton ME, Abu-Saad Huijer H, Geiger-Brown J: Fatigue, work schedules, and perceived performance in bedside care nurses. Workplace health \& safety 2017; 65: 304-312.

[28] Barker LM, Nussbaum MA: Fatigue, performance and the work environment: A survey of registered nurses. Journal of advanced nursing 2011; 67: 1370-1382. 